\title{
Le spectre de l'Orient dans les livres de famille florentins (1300-1480)
}

Lo spettro dell'Oriente nei libri di famiglia fiorentini (1300-1480)

The Specter of Orient in Florentine Family Books (1300-1480)

\section{Élise Leclerc}

\section{OpenEdition}

\section{Journals}

Édition électronique

URL : http://journals.openedition.org/cei/2677

DOI : $10.4000 /$ cei. 2677

ISSN : 2260-779X

\section{Éditeur}

UGA Éditions/Université Grenoble Alpes

Édition imprimée

Date de publication : 5 novembre 2015

Pagination : 87-103

ISBN : 978-2-84310-308-7

ISSN : 1770-9571

\section{Référence électronique}

Élise Leclerc, "Le spectre de l'Orient dans les livres de famille florentins (1300-1480) », Cahiers

d'études italiennes [En ligne], 21 | 2015, mis en ligne le 01 janvier 2017, consulté le 27 mars 2021. URL : http://journals.openedition.org/cei/2677 ; DOI : https://doi.org/10.4000/cei.2677 


\title{
LE SPECTRE DE L'ORIENT \\ DANS LES LIVRES DE FAMILLE FLORENTINS (1300-1480)
}

\author{
Élise Leclerc \\ Université Grenoble Alpes
}

Si l'Orient et ses personnages hauts en couleur étaient présents dans l'imaginaire florentin, grâce aux nouvelles de Boccace notamment, s'il suscitait chez les pèlerins l'envie de raconter leur périple et avait également sa place dans les correspondances des marchands, force est de constater que les habitants de la cité du lys ne lui font qu'une place très limitée dans leurs livres de familler. Comment interpréter cette présence marginale? Est-elle due aux enjeux spécifiques de cette forme d'écriture privée? La situation évolue-t-elle à mesure de l'avancée de l'Empire ottoman? Le cas oriental est-il spécifique ou bien est-il cohérent avec le traitement dans les ricordi et ricordanze des événements advenant hors des murs de la cité?

\section{Une présence marginale}

Les livres de famille produits par les Florentins au fil des XIV et $\mathrm{XV}^{\mathrm{e}}$ siècles constituent, par leur nombre avant tout, un cas hors-norme dans le panorama des écrits du for privé occidentaux : même si une part conséquente

I. Sur les livres de famille, voir les synthèses de C. Cazalé-Bérard et C. Klapisch-Zuber, "Mémoire de soi et des autres dans les livres de famille italiens ", Annales HSS, vol. 59, n 4, 2004, p. 805-826 et R. Mordenti (dir.), I libri di famiglia in Italia, II, Rome, Edizioni di Storia e Letteratura, 200I, où l'on en trouve une définition succincte (p. I5) : "Un libro di famiglia è un testo memoriale diaristico, plurale e plurigenerazionale, in cui la famiglia rappresenta tutti gli elementi del sistema comunicativo instaurato dal libro, costituisce cioè sia l'argomento (o contenuto) prevalente del messaggio testuale, sia il mittente che il destinatario della scrittura, sia infine il contesto e il canale della trasmissione. "Sur la diffusion du Décaméron et de ses figures marquantes — tel le Saladin — dans la société florentine, voir C. Bec, Les livres des Florentins (1413-1608), Florence, Olschki, 1984, et R. Girardi, Raccontare l'Altro. L'Oriente islamico nella novella italiana da Boccaccio a Bandello, Naples, Liguori, 2012. Sur les pèlerins et leurs récits, voir F. Cardini, In Terrasanta. Pellegrini italiani tra Medioevo e prima età moderna, Bologne, Il Mulino, 2002; Id., Toscana e Terrasanta nel Medioevo, Florence, Alinea, 1982. 
d'entre eux n'a probablement pas traversé les siècles, quelque cent cinquante livres nous sont parvenus ${ }^{2}$, qui s'échelonnent assez régulièrement sur la période $1300-1480^{3}$. Si ces ouvrages ont un horizon commun - conserver voire augmenter le statut de la famille en transmettant toutes les informations jugées bonnes et utiles pour ce faire - les modalités de réalisation de ce dessein sont extrêmement variées, ce qui se traduit dans la grande diversité des notes qui trouvent place dans ces livres : informations comptables, états du patrimoine mobilier et immobilier, comptabilité des naissances, décès ou mariages advenant dans la famille, ou encore listes des offices communaux occupés par les membres du lignage, récits à caractère historique, recettes médicinales... Dans le cadre de cette enquête, n'ont été prises en compte que les lignes et pages ayant trait aux faits ou événements à caractère historique ou politique que les pères jugèrent bon de rédiger afin d'en confier la mémoire à leurs descendants. Cela représente un corpus de I 289 notes, de longueur variable, se rapportant à des faits advenus en un lieu et un temps identifiables. Si, afin de limiter l'impact des intérêts propre à tel ou tel scripteur sur l'analyse statistique, on ne considère que les faits ayant donné lieu à au moins deux témoignages distincts, le corpus compte alors 685 notes, relatant 240 événements spécifiques. Or dans ce corpus, les faits advenant en Orient sont

2. Pour un catalogue quasi exhaustif de ces livres - qui constituent le corpus de cette étude — voir F. Pezzarossa, La tradizione fiorentina della memorialistica, dans G. M. Anselmi, F. Pezzarossa et L. Avellini (éd.), La "memoria» dei mercatores. Tendenze ideologiche, ricordanze, artigianato in versi nella Firenze del Quattrocento, Bologne, Pàtron, I980, p. 4I-I49 et la bibliographie de C. Klapisch-Zuber, La Maison et le nom. Stratégies et rituels dans l'Italie de la Renaissance, Paris, EHESS, I990. Certains ouvrages étant encore aujourd'hui conservés dans des archives familiales difficiles d'accès, cette contribution ne saurait présenter un tableau exhaustif des témoignages privés concernant l'Orient qui ont pu être produits par les Florentins des $\mathrm{XIV}^{\mathrm{e}}$ et $\mathrm{XV}^{\mathrm{e}}$ siècles. Les quelques données statistiques présentées ont vocation à offrir au lecteur un ordre d'idée. Précisons également que, du fait de la variété des informations qui composent les livres de famille, les frontières du corpus ne sont pas aisées à établir : la Cronica de Benedetto Dei, où il relate ses voyages et son séjour en Orient (voir P. Orvieto, "Un esperto orientalista del '400: Benedetto Dei", Rinascimento, vol. 9, I969, p. 205-275 et B. Dei, La cronica dall'anno I400 all'anno I500, R. Barducci [éd.], Florence, Papafava, 1984), et le "Diario di Felice Brancacci ambasciatore con Carlo Federighi al Cairo per il Comune di Firenze» (D. Catellacci [éd.], Archivio storico italiano, vol. 8, I88I, p. I57-I88; 326-334) ont été écartés du corpus, car ils n'ont pas la famille comme cadre de référence.

3. Lannée 1300 constitue la borne de départ de cette enquête en raison du nombre trop faible (4) de livres antérieurs comportant des notes à caractère historico-politique qui nous sont parvenus. L'année I480 a été choisie comme borne finale de cette enquête, car elle correspond à la fin d'un cycle politique (Laurent de Médicis a réussi à résoudre à son avantage la crise qui a suivi la conjuration des Pazzi, installant durablement son pouvoir à l'intérieur de Florence comme dans la Péninsule, où la situation demeure globalement stable jusqu'en I494), à un moment charnière sur le plan culturel (qui coïncide avec l'essor progressif de l'imprimerie, qui a pu avoir un impact non négligeable sur l'évolution des pratiques d'écriture des Florentins en leur offrant une possibilité nouvelle de thésauriser et de transmettre des connaissances au sein de la famille), mais aussi à la fin de l'expérience florentine en matière de flotte commerciale gérée par la Commune (voir à ce sujet M.E. Mallett, The Florentine Galleys in the Fifteenth Century, Oxford, Clarendon Press, 1967, et A. Sapori, "I primi viaggi di Levante e Ponente delle galere fiorentine", Archivio storico italiano, vol. II4, I956, p. 69-9I). 
extrêmement sous-représentés : seule l'ambassade de Felice Brancacci et Carlo Federighi auprès du sultan d'Égypte (I422) est rapportée par deux témoignages ${ }^{4}$, tandis que même la chute de Constantinople (I453) ne fait l'objet que d'une seule brève notes.

Pourtant, la menace que représentait l'expansion de l'Empire ottoman — qui a tant marqué les sociétés occidentales ${ }^{6}$ — n'est pas totalement absente des livres de famille florentins. On la trouve en I42I — conjuguée à une menace plus large de la libertas florentine - dans une prédication aux accents prophétiques que consigne Salvestro Mannini (I384-après I429), avant d'y ajouter un commentaire marginal : "[non disse vero] E prima piu volte avea detto che l'Imperatore verrebbe d'Aquilone con gran gente d'arme e barberi, e turchi, e molto offenderebbe questa Comunità con uccisione e con fuoco ${ }^{7}$.» Un an plus tard, le même auteur recopie une lettre concernant cette fois spécifiquement la menace ottomane, en l'occurrence l'assaut de Constantinople de I422:

Copia di lettera d'Atene di Romania; scrive Nerozzo di Luigi Pitti a Bonaccorso Pitti; fatta a' Atene adi 8 di novembre 1422.

Il gran Turco è stato all'assedio in persona a' Gostantinopoli circa di 3 mesi, et avendo molto stretta la terra, l'Imperadore volea abbandonare la terra per campare la sua persona; disselo co'l popolo, dove si trovò il Patriarca di Gostantinopoli, il quale

4. B. del Corazza, Quaderni overo Libro. Diario fiorentino (I405-I439), R. Gentile (éd.), Rome, De Rubeis Editore, I99I, p. 67; G. Rucellai, Zibaldone Quaresimale, A. Perosa (éd.), Londres, The Warburg Institute, I960, p. 45. Cette ambassade coïncide avec la création de l'office des Consuls de la mer (I42I), appelés à gérer la nouvelle flotte florentine. Voir à ce propos C. Tripodi, «Viaggi di ambasciatori tra Firenze e Il Cairo nel XV secolo", Mélanges de l'École française de Rome - Moyen Âge, vol. I22, nº 2, 2010, p. 4II-440.

5. G. Rucellai, ouvr. cité, p. 53 : "Nel 1453 e del mese di giungnio fu la perdita di Gostantinopoli in Grecia, presa dallo inperadore de' Turchi, che fu gran perdita al Cristianesimo. Diciesi che vi vene a chanpo con dugiento migliaia di persone tra piè e chavallo."

6. Sur les conséquences de cette expansion et de la chute de Constantinople sur la société et la culture occidentale contemporaine, voir notamment A. Pertusi (éd.), La caduta di Costantinopoli. Testi, I-II, Milan, Mondadori, 1976; R. Schwoebel, The Shadow of the Crescent: The Renaissance Image of the Turk (I453-I5I7), New York, St. Martin's Press, 1967; L'Europa dopo la caduta di Costantinopoli: 29 maggio I453 (Atti del XLVI convegno storico internazionale, Todi, octobre 2007), Spolète, CISAM, 2008; J. Hankins, "Renaissance Crusaders: Humanist Crusade Literature in the Age of Mehmed II ", Dumbarton Oaks Papers, vol. 49, 1995, p. III-207; M. Meserve, Empires of Islam in Renaissance Historical Thought, Cambridge Mass., Harvard UP, 2008; G. Ricci, I Turchi alle porte, Bologne, Il Mulino, 2008.

7. Salvestro Mannini, Biblioteca Nazionale Centrale di Firenze, II. IV. 380, p. 428. Il s'agit d'un manuscrit contenant les notes de lecture prises par le sénateur Carlo Strozzi à partir de l'original, dont la trace a été perdue. Comme à son habitude, Strozzi recopie les extraits qui l'intéressent et indique à la fin du paragraphe le folio du manuscrit original dont est tiré le passage (ici, fo 58 ). Les critères de transcription adoptés sont ceux communément employés pour l'édition de livres de famille (voir F. Castellani, Ricordanze A (I436-I459), G. Ciappelli [éd.], Florence, Olschki, 1992, p. 57-59; A. Molho et F. Sznura, «Brighe, affanni, volgimenti di stato". Le ricordanze quattrocentesche di Luca di Matteo di messer Luca dei Firidolfi da Panzano. Florence, SISMEL-Edizioni del Galluzzo, 20IO, p. CII-CIII). Sont indiquées par $<\ldots>$ les extrapolations liées à l'état du manuscrit ou de l'écriture. Les interventions éditoriales sont présentées entre crochets. Les abréviations courantes ont été résolues sans indication typographique. 
fù il primo che rispuose, dicendo che ciò non gli parea che dovesse fare, e che per avere della fede Christiana, egli volea essere alla battaglia il primo morto, ma che egli sperava in Dio, che per lui, e per quello popolo provederebbe a salvare quella Città, allora l'Imperadore disse essere contento di rimanere alla difesa di quella Città, se bene fusse certo d'essere morto, il perché quello popolo ne prese conforto, gridando battaglia i Turchi che già aveano rotto il muro del primo cerchio, e del secondo, vennono al terzo cerchio allora i Greci uscirono alla battaglia, e per forza cacciarono fuori i Turchi de quali vi rimasono morti circa a Is mila, e de Greci furono morti circa a 4 mila, nientedimeno i Turchi feciono gran' pugna però credettono pigliare tutta la Città, e avendo il Gran Turco avuta detta rotta si partì et allunghòssi da Gostantinopoli ben tre giornate; la cagione della vettoria de Greci fù perché in Gostantinopoli si trovaron' circa di 4 mila balestrieri Italiani, i quali furono quelli che difensono la Città, E rimasa assediata Salonichi, e'l Sitone terre dell'Imperadore, e anche di nuovo il Turco manda molti Turchi al Sitone, perché quivi anche à ricevuta gran' rotta sua gente ${ }^{8}$.

Dans cette lettre, l'accent est mis sur l'action des chrétiens face aux Turcs d'une part, et d'autre part sur le rôle — crucial — des Italiens dans l'événement. Or ces deux éléments sont également présents dans le récit de la bataille de Belgrade (1456) que rédige Francesco di Tommaso Giovanni (I4O2-I458) :

\section{El turco rotto da' Cristiani}

A dì ${ }^{9}$ Io d'agosto 1456 venne in Firenze l'aviso come per divina grazia el grande turcho, con infiniti esserciti è stato rotto da' Cristiani; cioè a dì ***, l'armata nostra gli ruppe in mare e l'altro dì Giovanni [Hunyadi] v<aiv>oda, capitano generale degl'Ungheri e d'altre nationi di là gli ruppe per terra con infinita ucisione de' loro e molti cristiani, e fu cosa miracolosa la grande e infinita strage che dicono esservi stata, inperò che l'asaltorono al suo padiglone, e con maxima vergogna e viltà sua si fugì con circa 300 cavalli. Dissono poi certi di loro, grandi signori che resterono prigioni, che vedevano infinita multitudine contro a.lloro, onde si stima esser stato cosa miracolosa e divino adiutorio, inperò che e nostri furono picolissimo numero rispetto a.lloro, e martedì sera a dì x si fece grandissima festa di fuochi e campane ecc. Di poi si feciono più processioni fino a domenica che si fé una solenne con maxima devotione ${ }^{\mathrm{IO}}$.

Ce que l'auteur estime bon et utile d'enregistrer, c'est une victoire de la Chrétienté face aux Ottomans, la victoire d'une communauté à laquelle il s'identifie (à travers le pronom possessif «les nôtres», qui désigne le camp chrétien) et dont il prend soin de signaler qu’elle était composée de

\footnotetext{
8. Ibid., p. 435 [= fo 68$]$.

9. primo barré.

Io. Francesco di Tommaso Giovanni, Archivio di Stato di Firenze [infra ASF], Carte strozziane, II, I6 bis, fo $23 \mathrm{r}$.
} 
Hongrois, certes, mais aussi d' "autres nations», parmi lesquelles il est aisé d'imaginer les Italiens.

Un certain tropisme italien, sinon florentin, semble ainsi présider à l'entrée dans l'écriture privée d'événements survenus dans des contrées plus ou moins orientales : Donato Velluti n'aurait ainsi probablement pas évoqué la nomination de l'archevêque de Patras en Roumanie (I358) s'il ne s'était pas agi du rejeton d'un grand lignage florentin qui comptait parmi sa suite un membre de la propre famille de l'auteur ${ }^{\mathrm{II}}$. Ce qui compte à ses yeux, c'est la réussite de son parent, qui s'était enrichi avant de mourir au loin, malgré la menace que représentaient déjà les Turcs, évoqués seulement "en passant». De même, si Naddo da Montecatini évoque Rhodes, c'est parce qu'un illustre compatriote y mourut en $1388^{\mathrm{I2}}$. Et Bonaccorso Pitti ne présente pas la victoire ottomane contre la France lors de la bataille de Nicopolis (1396) comme un événement en soi, digne d'être enregistré comme tel, mais seulement comme un contretemps ayant ralenti la bonne exécution de sa mission d'ambassadeur, qui est la motivation première du récit ${ }^{\mathrm{I}}$.

Ainsi, dans les rares cas où les Florentins jugent digne de faire entrer dans le livre de famille un fait survenu dans des contrées orientales, il semble que ce soit dans le but de mettre en valeur le rôle joué dans les événements par une communauté envers laquelle l'auteur nourrit un senti-

II. D. Velluti, La cronica domestica di messer Donato Velluti scritta fra il 1367 e il 1370 con le addizioni di Paolo Velluti, scritte fra il I55s e il I560, I. del Lungo (éd.), Florence, Sansoni, I9I4, p. 35 : "Poi essendo stato una pezza di qua, venne caso che messer [Giovanni] di Iacopo di Donato Acciaiuoli fu fatto arcivescovo di Patras in Romania: onde andando molti giovani di Firenze collui, v' andò anche il detto Gherardino, e lui servi mentre che vivette, avvegnadio che poco vivesse di là; ma in quello tempo gli fu assai innanzi, e da lui era volentieri veduto; e se fosse vivuto, i fatti suoi sarebbono stati bene, però che gì̀ alla sua vita avea fatto avanzo di certa quantità di danari, che che a grandi rischi fu più volte per zuffe fatte tra loro e' Turchi."

I2. Naddo da Montecatini, Memorie storiche da un libro di ricordi scritto da Naddo di ser Nepo di ser Galo da Montecatini di Valdiniveole cittadino Fiorentino. Dall'anno 1374 all'anno 1398, I. di San Luigi (éd.), Delizie degli eruditi toscani, XVIII, Florence, Cambiagi, 1784, p. 99 : "A di primo d'aprile venne novelle a Firenze, che messer Benedetto, ed Agnolo suo nipote degli Alberti mori a Rodi."

13. B. Pitti, "Ricordi", dans V. Branca (éd.), Mercanti scrittori, Ricordi nella Firenze tra Medioevo e Rinascimento, Milan, Rusconi, 1986, p. 406-408: "Trovai che lo Re era forte malato e trovai che v'erano venute le vere novelle della sconfitta de' Francieschi auta in Turchia, per le quali due cagioni io pote' poco adoperare innanzi che messer Vanni e messer Filippo Corsini v'arrivassono. Vennono e con messer Filippo venne Luigi mio fratello. Stemmo circa di 4 mesi, che quivi non s'attendea se non a fare esequi di gran signori reali e altri morti in Turchia, e lo Re malato e rinchiuso come folle. Avvenne la sua guarigione per modo che cominciò a entrare in consiglio. [...] Fececi chiamare dentro e il cancelliere ci rispose in questo effetto, imprima scusando lo Re del non averci più tosto data la risposta e fatto l'effetto della nostra domanda, assegnando con oneste parole la cagione della sua malattia e appresso per lo caso de' suoi congiunti morti in Turchia, ma che egli era disposto fare verso di noi il suo dovere." Pour les motivations de l'écriture de Pitti, voir A.-C. Fiorato, "Propedeutica e legittimazione al potere nei "Ricordi" di Bonaccorso Pitti", dans L. Secchi Tarugi (éd.), Cultura e potere nel Rinascimento (Atti del IX Convegno internazionale, Chianciano-Pienza, 1997), Florence, Franco Cesati Editore, p. 19-32 et L. Pandimiglio, "Pigliate esempro di questo caso: l'inizio della scrittura di Bonaccorso Pitti", Lettere italiane, vol. 40, nº 2, I988, p. I6I-I73. 
ment d'appartenance (la famille, les Florentins, les Italiens, la Chrétienté). Ce rôle de faire-valoir rejoint donc la stratégie générale qui préside à l'écriture des ricordi et ricordanze, à savoir conserver et transmettre la mémoire de tous les éléments pouvant conforter voire augmenter le statut et le rayonnement de la famille.

Cela se vérifie dans les notes - à peine plus nombreuses - qui ont trait à des faits qui se déroulent à Florence ou en Italie mais concernent l'Orient. Trois auteurs évoquent ainsi la venue à Florence de l'empereur Jean VIII Paléologue en février I439, dans le cadre du concile de Florence : Bartolomeo del Corazza (I38I-I449), Giovanni de' Pigli (I396-I473) et Giovanni Rucellai (I403-I48I). Le récit du second est particulier car celuici reçut l'empereur sous son propre toit : hormis quelques remarques sur les mœurs exotiques du souverain étranger, son propos est entièrement placé sous le signe de l'honneur que ce séjour représenta pour lui et sa famille ${ }^{\mathrm{I}}$. Simple spectateur de l'événement, Bartolomeo del Corazza se borne à enregistrer les honneurs faits et reçus par la Commune à l'occasion de l'entrée de l'empereur d'Orient ${ }^{15}$, tandis que Giovanni Rucellai

I4. G. De’ Pigli, dans K. M. Setton (éd.), "The Emperor John VIII slept here», Speculum, vol. 33, n 2, I958, p. 225-226: "[...] messere Angnolo di Jachopo Acciaiuoli [...] [r] ispuosemi: io vengho da Pistoia et da Prato in chompagnia dello imperadore di Chostantinopoli, il quale volle andare a vedere Pistoia e lla Cintola di nostra Donna a Prato, e io gli fu dato in chompagnia dalla Signoria; et perché l'ora è tarda a irne in Firenze a mangiare, e lui e stracho et infermo, chome sai, volevo alloggiarllo in questa chiesa per insino a stasera. Io gli rispuosi: messere, io sono qui a chasa nostra e sonci solo; se quella fosse sofficiente a ricettare tanto signore, mi sarebbe singhulare piacere avisandovi [Iogr] che in essa nonne se non letta e'l ghuscio della chasa. [...] Et quanto pote mi ringraziò et acciettò di chondurllo a chasa nostra chon dirmi: io non voglio tu ci metta niente del tuo se non la brigha della chasa, che lle spese gli fa il Chomune. E tornossi verso Prato, dove lo 'mperadore fra via rischontrò, et diritto a chasa nostra lo chondusse, nella quale venne chon quaranta in cinquanta chavagli molto bene a punto et chon molti suoi baroni, signori e gentili huomini; [...]. Io gli avevo fatto aparechiare il letto di chamera a man mancha a entrare nella nostra sala [...]. Lui, sechondo compresi, non vi volle entrare, anzi si fecie fare in su due panche uno letucio cho uno materassino et chon uno tapeto allato all'uscio della sala, a man sinistra allo entrare dentro, sotto la perghola; e quivi dormi uno sonno per insino che quegli suoi providono al suo mangiare. Et provisto ch'ebono, si fecie porre una tavoletta inanzi a quello lettucio, et io gli trovai delle tovaglie bianche; et quivi mangio solo, et gli altri suoi baroni e signori sotto la perghola et di fuori et dentro, chome alla sachomanna. [...] Et nota, chella prima vivanda mangiò fu una insa [I0gv] lata di porcellana et di prezemoli chon delle cipolle, et lui stesso la volle nettare. Dipoi ebono pollastri e pipioni lessi, e dipoi pollastri e pipioni squartati e fritti nella padella chon lardo. Et chome venivano le vivande, tutte gli eran poste davanti, et lui prendeva quello voleva, et mandava agli altri suoi; e ll'ultima sua vivanda fu certe huova gittate in su i mattoni chaldi, dove serano chotte l'altre chose; e messogliele in una schodella chon di molto spezie, non so inmaginare chome si eran fatte, ma chosi fu il vero. [...] Messere Angnolo et Ciriacho d'Anchona, huomo dottissimo in grecho et in latino, e io ci stemo tutto giorno per lla sala, lui sempre giuchando a tavole et motteggiando chon quelli suoi. In sulla sera, a ore xxiii, e per ventura piu tardi, messere Angnolo mandò per me, ch'ero nell'orto chon quelli suoi gientili huomini, et feciemi inginiochiare ai piedi del detto imperadore; lui mi fecie ringraziare dal suo interpito dell'onore [the words delle nostre are here struck out] che io gli avevo fatto del riceverllo in chasa, et fattomi proferere, semai chapitassi ne suoi terreni, mi farebbe onore, [IIor] ec., e prese il nome mio, et chome si chiamava, dove era stato et feciene fare nota. [...] Et noi dipoi, a chonmemorazione delle sudette chose, faciemo dipignere l'arme sua di sopra l'uscio della nostra sala, chome anchora si vede."

I5. B. del Corazza, ouvr. cité, p. 36-37. 
souligne le rôle crucial joué par le chancelier de Florence Leonardo Bruni dans l'avancement des discussions ${ }^{\mathrm{I}}$.

Si les notes spécifiquement dédiées aux affaires orientales n’abondent toujours pas après $1453^{17}$, on constate cependant que le Grand Turc fait régulièrement figure d'épouvantail pour la communauté chrétienne, un épouvantail que les pontifes n’hésitent pas à brandir afin de resserrer les rangs de leurs ouailles. En octobre I455, Francesco di Tommaso Giovanni choisit ainsi de conserver la mémoire d'une procession explicitement organisée pour implorer l'aide divine contre les Ottomans :

\section{Le processioni per l'aiutorio di dio contro al turco}

Ricordo che giovedì a dì 16 d'ottobre $\mathrm{I} 455$, Cominciorno a farsi le processioni solenni per impetrare l'aiutorio divino contro al turco e iiij giorni, cioè ogni dì a jo quartiere, sempre multiplicò il popolo, e da prima e fanciulli e fanciulle poi in ultimo le donne assai vestite di bianco, cioè con camice sopra e panni et $\mathrm{j}^{\mathrm{a}}$ croce rossa nel petto, e più $\mathrm{e}$ più giorni da prima predicò in Santa Maria del Fiore maestro Giovanni da Napoli de frati predicatori, commessario e generale mandatario del papa con plenissima autorità apostolica in disporre e concedere qualunque cosa in favore e susidio de cristiani contro al turco per difensione della fede. Per lo simile modo fu ordinato per l'altre città d'Italia ${ }^{18}$.

En I459, Bernardo Rinieri ressent le besoin de préciser à l'occasion de son récit de l'entrée du pape Pie II à Florence que celui-ci se rendait à Mantoue "pour y prendre des mesures contre le Turc ${ }^{19}$ ", motif qu'il répète, quelques mois plus tard, lorsqu'il enregistre la nouvelle visite du pontife

I6. G. Rucellai, ouvr. cité, p. 48 : "[...] nel 1439 ritornò in Firenze insieme collo inperadore de' Greci e stetteci sino nel I442. E in detto tenpo si conchiuse in Firenze l'unione de' Greci della diferenza che era della fede, et simile l'unione degli Ermini con belle et grandi disputazioni. Et fu oppinione che l'opere di messer Lionardo d'Arezo, poeta dottissimo in grecho e i.llatino e in ebraicho, fosse chagione di disporre i detti Greci interamente alla fede della Chiesa romana; il quale messer Lionardo era i quel tenpo chancielliere della singnioria di Firenze."

I7. Dans le livre privé de Neri di Bicci — à mi-chemin entre le carnet professionnel et le livre de famille on trouve un écho très indirect de la chute de Constantinople, à l'occasion d'une commande spéciale de la Seigneurie de Florence : "MCCCCLIIII. A dì Is d'aghosto I454. Tabernacholo fe' a' Signiori nel'Udienza. Richordo che detto dì Is d'aghosto io Neri di Bicci dipintore tolsi a mettere d'oro e dipigniere uno tabernacholo di legniame fatto al'anticha [...], il quale à stare d'atorno a uno armare dove istano le Pandette e uno altro libro, il quale vene di Ghostantinopoli e certe altre solenisime chose del Popolo di Firenze [...]. " (Ricordanze segnato D, B. Santi [éd.], Pise, Marlin, I976, p. I7)

I8. F. di T. Giovanni, ouvr. cité, fo 2 Ir.

19. K. Colin, Le Ricordanze di Bernardo Rinieri - edizione, [thèse de laurea autorisée à la consultation, dir. C. Bianca], Università di Firenze, 2002, p. $55: "+A$ dì 25 di aprile, in mercholedi [Ent.a di Papa Pio in Fire.] Richordo come questo sopradetto di, entrò in Firenze il Papa, e chiamavasi Papa Pio de Picholuomini da Siena, con ro chardinali e fecesi portare in sun'una sedia dal signore Gismondo da Rimino e il signore Astore da Faenza e il signore di Frulli e altri signori di Romagna. E il chonte Ghaleazo, di sopra detto, era a chavallo a'llato a'llui, da'llato destro. Andava a Mantova per far provedimento contra 'l Turcho, sechondo si diceva. Di poi sabato a di 5 , parti per Mantova, coé a di s di maggo." 
à son retour de Mantoue ${ }^{20}$. À l'occasion de son récit de la mort de Côme de Médicis en I464, Laurent le Magnifique dresse un état des lieux de la Péninsule, en paix — notamment grâce à l'action de son aïeul — à l'exception des Vénitiens, "en guerre contre le Turc» et souligne lui aussi l'implication du pape Pie II dans cette lutte ${ }^{21}$. En 1476 (I475 selon le style florentin), ce conflit est toujours présent dans les consciences florentines :

A dì I2 di marzo I475 andò Jachopo mio figliuolo chol veschovo di Buda d'Ungheria a Roma, el quale andava per inbasciadore al papa e altri Taliani chon uno altro inbasciadore per parte del ducha Istefano vaivoda, chapittano chontra agli Turchi; <e v'era venuto $>$ da' Taliani per avere denari et soldare giente che l'aspetta questa istate. E l'an[n]o pas[s]ato fu alle mani cho.Turchi detto ducha ed ebbe grande vittoria; che chosì l'abbia per llo avenire ${ }^{22}$.

Les éléments précédemment identifiés sont encore une fois présents : la famille est directement impliquée dans la lutte, présentée comme organisée autour du pape mais impliquant aussi l'ensemble des Italiens. Notons également que l'auteur revient sur la victoire obtenue l'année précédente par le capitaine des armées occidentales, inscrivant ainsi l'entreprise dans une dynamique positive pour la communauté à laquelle il appartient. Quelques années plus tard, sous la plume de Filippo Strozzi (I428-I49I), le Grand Turc constitue toujours une menace que le pape n'hésite pas à brandir pour faire filer droit ses ouailles : l'ambassade florentine envoyée à Rome en novembre I479, dans le cadre des suites de la conjuration des Pazzi obtient ainsi le pardon du pape pour la communauté florentine en échange de l'envoi de quinze navires pour combattre les Ottomans ${ }^{23}$.

20. Ibid., p. 6I : "+ Domenicha a di xxvii di gennaio [entrata di Papa Pio] Richordo come questo sopradetto di, entrò il Papa Pio in Firenze e venia da Mantova, dove era stato da maggo prima fino ad ora, per fare chonchrusione dell'ampresa del Turcho. E tutti i signori christiani vi mandarono ambasciatori. Entrò in Firenze con s chardinali. Di poi si parti martedi a di xxviiijo di gennaio, circha ore I6."

2I. M. Del Piazzo (éd.), "Gli autografi di L. De' Medici nell'Archivio di Stato di Firenze", Rinascimento, vol. 8, 1957, p. 222 : "A dì primo di agosto morì Chosimo de' Medici el quale lasciò la città sua 'n pace dentro e di fuori e in grande habundanzia di grano e in magiore felicità che lla fusse mai stata; stavano le potentie d'Italia chome da piè dirò che la Italia era divisa in s potentie benché fussino molte più pure quelle di che si facea conto erano 5, papa, Re di Napoli, Signoria di Vinegia, duca di Milano e Fiorentini. El papa era in quel tempo di natione Sanese chiamato Pio II, re di Napoli el re Ferdinando figluolo non legittimo del re Alphonso, el doge di Vinegia era messer Cristofano Moro et duca di Milano el duca Francesco Sforza, quell'altre tre potentie si reggevano e reggono a popolo; Firenze si regge a popolo; le quale tutte erano [ancora cancellato] insieme in lega e stavano in pace eccepto e' vinitiani che erano e sono in guerra col turco. Alla quale impresa volendo el papa dare favore etc. havea presso Adrie convocate certe potentie di Italia e fuora d'Italia che concorressino a tale impresa e per essere in luogo più vicino alle terre del Turco, foe Capogrosso nel porto d'Ancona dove lui medesimo et gran parte de' cardinali convennono et similmente vi venne il doge di Vinegia con galee armate."

22. Giovanni di Guido Baldovinetti, ASF, Acquisti e Doni, I90/3 (II), fo 3Ir.

23. F. Strozzi, ASF, Carte Strozziane, V, 22, fo IO6v : "E del mese di novembre mandamo a Roma II inbasciadori a domandar perdono, e insieme chon messere Antonio Ridolfi che v'era doma[n]dorono perdono per tutti e ciptadini e suditti e dopo alchuni dì, e a porta della chiexa serrati, in domenicha a dì 3 di dicembre perdonò $e$ 
Ainsi, alors que plusieurs études ont mis en avant la frilosité des Médicis à l'égard de la lutte contre l'Empire ottoman voulue par le pape, puis la politique de rapprochement avec Mehmed II mise en ouvre par Laurent de Médicis ${ }^{24}$, il apparait que chez les auteurs de livres de famille, c'est le motif de la "menace turque», tel qu’il fut véhiculé par les autorités religieuses en particulier, qui prime.

Du point de vue chronologique, il est également frappant de constater que le - très relatif - essor des notes concernant l'Orient coïncide avec la mise en place d'une politique communale de navigation commerciale longue distance 25 - I422 concentrant trois des cinq notes de ce type que contient l'ensemble du corpus. Cet engagement étant le fait de la classe dirigeante florentine, à une époque où la vision du stato — le statut — des familles n'était pas encore distincte du stato de la cité — appelé à devenir l'État - les notes de ce type peuvent alors servir le même dessein que les autres informations à caractère historique ou politique contenues dans les livres privés de ces familles, à savoir transmettre aux descendants la mémoire de faits pouvant leur servir à conserver ou augmenter le stato de la famille ${ }^{26}$. Le fait que ces quelques notes soient marquées par l'honneur ou les honneurs que l'auteur retire des faits relatés, en tant que membre d'une communauté — la famille, Florence, l'Italie, la Chrétienté — irait dans le sens de cette interprétation. Pour marginale qu'elle soit, la présence de l'Orient dans les livres de famille florentins serait donc cohérente avec les stratégies traditionnellement mises en œuvre en leur sein. Reste cependant, afin de conforter cette hypothèse, à mesurer la spécificité au sein du corpus des notes traitant de l'Orient par rapport à celles qui ont plus généralement trait à ce qui se passe hors des murs de la cité.

ribenedi, dandoci per penitenzia d'armare per questo anno Is ghalere chontro al turcho." Ce livre a fait l'objet d'une édition partielle: G. Capponi, Storia della Repubblica di Firenze, II, Florence, Barbèra, I875, p. 520-52I.

24. Voir F. Babinger, "Lorenzo de' Medici e la Corte ottomana», Archivio storico italiano, vol. I2I, I963, p. 305-36r ; L. Tanzini, «Il Magnifico e il Turco. Elementi politici, economici e culturali nelle relazioni tra Firenze e Impero Ottomano al tempo di Lorenzo de' Medici», RiMe, ${ }^{\circ}$ 4, 20IO, p. 27I-289, et pour les relations entre les autorités florentines et l'Orient: G. Müller, Documenti sulle relazioni delle città toscane coll'Oriente cristiano e coi turchi fino all'anno MDXXXI, Florence, Cellini, I879.

25. Sur ce point, outre les ouvrages cités de M. Mallett et A. Sapori, voir B. Dini, «L'economia fiorentina dal I45O al I530", dans Saggi su una economia-mondo. Firenze e l'Italia fra Mediterraneo ed Europa (secc. XIII-XVI), Pise, Pacini, I995, p. I87-2I4 et H. Hoshino, «I mercanti fiorentini ad Alessandria d'Egitto nella seconda metà del Trecento", dans Industria tessile e commercio internazionale nella Firenze del tardo Medioevo, F. Franceschi et S. Tognetti (éd.), Florence, Olschki, 20oI, p. IOI-II2; Id., «Il commercio fiorentino nell'Impero Ottomano: costi e profitti negli anni I484-I488", dans Industria tessile..., ouvr. cité, p. II3-I23 et Id., L'Arte della Lana in Firenze nel Basso Medioevo. Il commercio della lana e il mercato dei panni fiorentini nei secoli XIII-XV, Florence, Olschki, I980.

26. Sur ce point, que je ne peux développer ici, qu'il me soit permis de renvoyer à mon travail de thèse (Affaires de familles et affaires de la cité. La transmission d'une pensée politique dans les livres de famille florentins, $X I V^{e}-X V^{e}$, ENS de Lyon, dir. J.-C. Zancarini, 20I3). 


\section{L'ici et l'ailleurs dans les livres de famille florentins}

Sans grande surprise, les notes se rapportant à des faits advenus à Florence sont bien plus nombreuses dans le corpus que celles ayant pour cadre de référence la Toscane, la péninsule italienne ou l'étranger (fig. Ia et fig. Ib). Notons que si les proportions des notes concernant Florence sont sensiblement les mêmes selon que l'on considère l'ensemble du corpus ou uniquement les événements rapportés par au moins deux auteurs distincts, il n'en va pas de même pour les autres catégories, et en particulier les notes concernant l'étranger (qui représentent respectivement $2 \%$ ou $4 \%$ ). Les expériences et intérêts personnels des scripteurs influent donc manifestement sur leur motivation - ou non — à prendre la plume pour traiter d'événements survenant hors des murs de la cité.

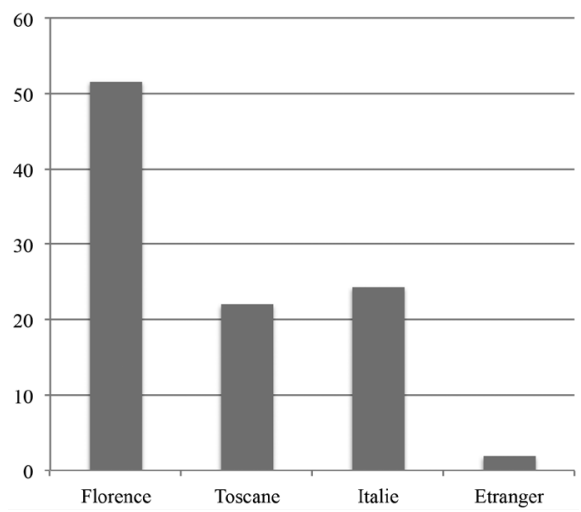

Fig. Ia. - Répartition des lieux de référence des notes du corpus restreint (en pourcentage).

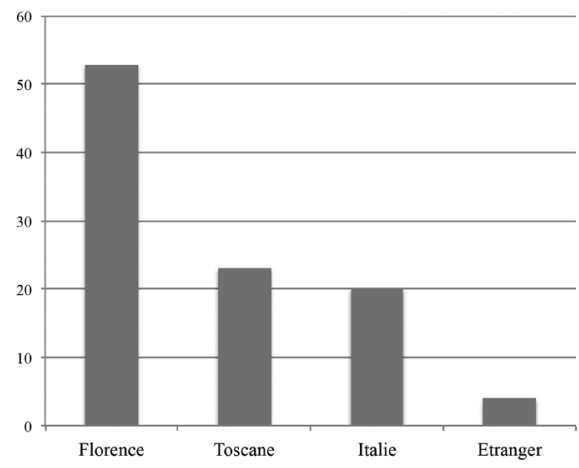

Fig. Ib. - Répartition des lieux de référence des notes du corpus total (en pourcentage). 
Si l'on examine la répartition des notes ayant Florence, la Toscane, l'Italie ou l'étranger comme cadre de référence au fil du temps (fig. za et fig. $2 b^{27}$ ), on voit que la surreprésentation de Florence perdure, à l'exception notable de la période de rédaction I40O-I4IO, ce que l'actualité de l'époque (la guerre contre Milan, la prise de Pise...) peut en partie expliquer ${ }^{28}$. Mais le début du $\mathrm{Xv}^{\mathrm{e}}$ siècle est aussi marqué par une notable augmentation des notes concernant l'Ailleurs, quelle que soit la taille du corpus considéré. Alors que les affaires italiennes ou étrangères n'attiraient manifestement pas l'attention des Florentins - sauf cas particuliers, liés à des intérêts personnels ou familiaux — jusqu'en I340, l'Italie s'impose toujours plus comme un cadre de référence, au détriment de la Toscane, chez les auteurs du $\mathrm{Xv}^{\mathrm{e}}$ siècle. Difficile de tirer davantage de conclusions de ces statistiques : les variations importantes entre les pourcentages — selon que l'on considère le corpus total ou le corpus restreint aux faits rapportés par deux auteurs ou plus - sont en effet une invitation à entrer dans l'analyse qualitative.

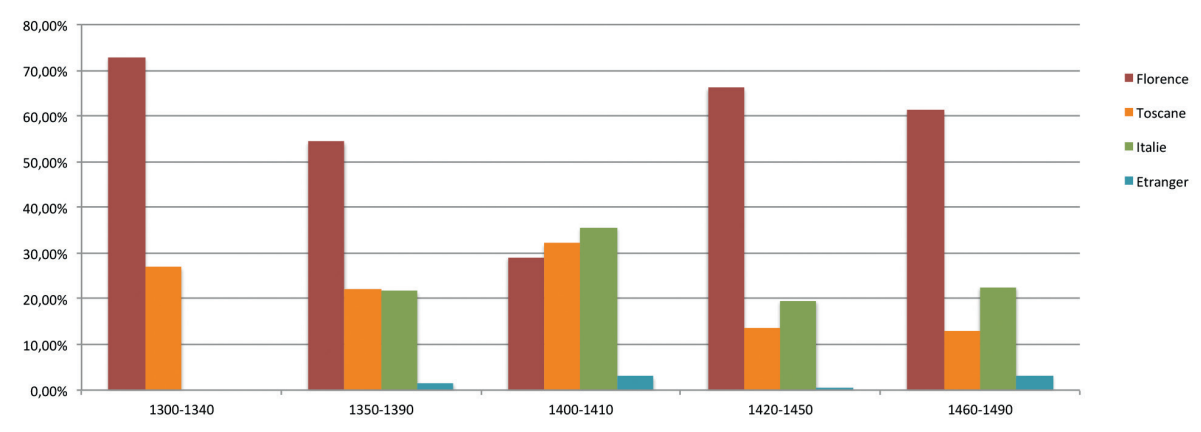

Fig. 2a. - Portée du regard des Florentins au fil du temps

(dans le corpus restreint).

27. Une décennie de référence a été associée à chaque livre, qui correspond à la valeur médiane calculée à partir des dates de début et de fin de rédaction. Les périodes indiquées sur les graphiques résultent d'un regroupement arbitraire - et donc certainement discutable — issu d'une tentative de former au sein du corpus des ensembles comparables du point de vue du nombre de notes rédigées durant la période (en l'occurrence, pour le corpus restreint : 38 notes pour la période I300-I340, I93 pour I350-I390, I99 pour I40O-I4IO, I63 pour I42OI450, 93 pour I460-I490). Je tiens à remercier T. Vuillaumier pour son aide dans l'élaboration des graphiques présentés ici.

28. À moins que l'on ne veuille y voir une manifestation des changements intervenus dans la pensée politique florentine durant ce qu'Hans Baron a nommé l'«humanisme civique». Cette question n'étant pas directement liée au propos de cet article — nous n'entrerons pas dans ce débat —, voir notamment : J. Hankins, Renaissance Civic Humanism: Reappraisals and Reflections, Cambridge, Cambridge UP, 200o; L. Baggioni, La Forteresse de la raison. Lectures de l'humanisme politique florentin d'après l'Epistolario de Coluccio Salutati (I33I-I406), thèse de doctorat (dir. J.-C. Zancarini), ENS de Lyon, 201 (à paraître chez Droz). 


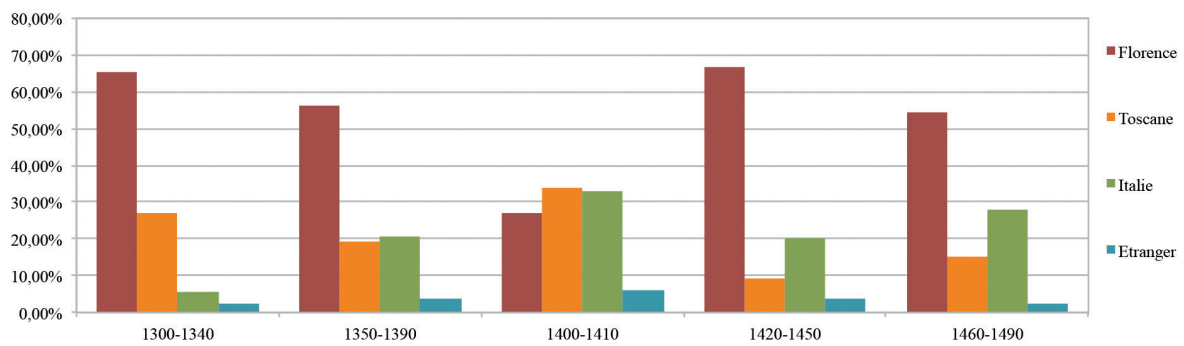

Fig. 2b. - Portée du regard des Florentins au fil du temps

(dans le corpus total).

Un examen plus attentif des notes concernant les événements advenant hors de la Péninsule montre qu'elles sont avant tout focalisées sur les deux puissances supra-étatiques ante litteram que furent l'empire et la papauté (durant le schisme notamment), puissances dont l'action avait des conséquences notables et directes sur la vie politique florentine ${ }^{29}$. Les quelques notes liées à la dynastie d'Anjou-Duras — dont le destin fut aussi très lié à la Péninsule — peuvent également être reliées à cette catégorie de notes qui concernent nominalement l'«ailleurs" mais ont impact direct ou presque sur Florence ${ }^{30}$. Les notes qui concernent le destin des Florentins à l'étranger forment quant à elles une deuxième catégorie, dont le lien avec l' "ici», et en particulier le stato des familles florentines, est également très net ${ }^{3 \mathrm{I}}$.

29. Sur les 39 notes concernant des faits survenus hors de la Péninsule que comprend l'ensemble du corpus, onze ont trait aux affaires impériales, sept à celles de la papauté, ce qui représente près de la moitié du total. Les notes les plus anciennes (avant I350) concernent uniquement l'Empire (Neri degli Strinati relate le couronnement de l'empereur Henri VII en I3ı2; Simone della Tosa, l'excommunication de l'empereur Louis IV de Bavière en 1324), tandis qu'à partir des années 1360 les affaires de la papauté hors d'Italie apparaissent aux côtés des affaires impériales (l'ambassade de Brunetto Latini aux empereurs concurrents de 1258 ainsi que l'ambassade des Guelfes à Curradino en I26I sont rappelées par Lapo da Castiglionchio à la fin des années I37O, à partir de la Chronique de Giovanni Villani; l'ambassade de 1352 à l'empereur est relatée par Donato Velluti, qui écrit à la fin des années 1360, et Agnolo de' Ricci, qui écrit dans les années I460; le retour en Avignon d'Urbain V en I370, sa mort et l'élection du pape Grégoire XI par Guido Monaldi et l'ambassade à ce pape en 1373 par Bese Magalotti, l'ambassade à l'antipape Benoît XIII de 1395 par Bonaccorso Pitti, l'élection au siège impérial de Robert de Bavière en I4OI et l'ambassade florentine qui lui fut ensuite envoyée par Giovanni Morelli et Jacopo Salviati, la nouvelle ambassade à Benoît XIII dans le cadre du schisme, par Jacopo Salviati, l'élection de Martin V en I4I7, par Doffo Spini, l'annonce de la possible venue de l'empereur en Italie en I422, par S. Mannini).

30. Le couronnement de Charles III de Duras en Hongrie, puis son assassinat et la capture de la reine (I386), sont rapportés par Naddo da Montecatini, tandis que le couronnement de Ladislas Ir d'Anjou-Duras en Hongrie (1403) est relaté par Luca Buondelmonti.

3I. Outre la nomination de l'archevêque Acciaiuoli à Patras (I358) et la mort de Benedetto Alberti (I388), déjà citées, cette catégorie comprend l'enregistrement par G. Monaldi de la mort d'un illustre concitoyen, Luigi Gianfigliazzi, en Avignon en 1371, la tentative en I38I d'obtenir du roi d'Angleterre la libération d'otages 
Parmi les trente-neuf notes concernant des faits advenus hors de la Péninsule dans l'ensemble du corpus, dix-sept se rapportent à des ambassades envoyées par la Seigneurie florentine, ce qui démontre encore une fois la force du lien entre ce qui est jugé digne d'entrer dans le livre de famille et ce qui se passe au sein des murs de la cité. Dans cet ensemble, on peut cependant distinguer entre les huit récits émanant d'un auteur directement impliqué dans l'ambassade — parce qu'il fut lui-même ambassadeur, ou parce que l'ambassadeur fut un membre de sa famille ${ }^{32}$ - et les ambassades rapportées par des auteurs n'ayant rien à voir dans les événements, dont on peut considérer qu'elles constituaient pour eux des faits politiques majeurs, dignes d'être consignés en tant que tels. Parmi ces neufs notes, quatre concernent les affaires impériales ${ }^{33}$, trois le royaume de France ${ }^{34}$, et deux le sultan d'Égypte ${ }^{35}$. La participation d'un membre de la famille aux événements semble donc encore une fois déterminer largement l'entrée ou non d'un fait dans le livre privé, ce qui est cohérent avec les enjeux et les horizons de cette pratique d'écriture; et même quand ce n'est pas le cas, le lien avec les affaires de la cité demeure bien souvent très net. Les quelques notes restantes le confirment: Bonaccorso Pitti et Salvestro Mannini sont très préoccupés par les conflits opposant la France à l'Angleterre en raison de liens et d'intérêts personnels vis-à-vis de ces pays ${ }^{36}$. Quant aux trois notes mentionnant la signature d'une alliance entre la France et Florence contre Milan en 1396, le lien avec les affaires — pressantes — de la cité est manifeste ${ }^{37}$. En somme, seules les quelques notes qui ont trait à l'Orient — l'assaut à Constantinople de I422, la chute

florentins, relatée par B. Pitti, des mesures prises contre les marchands florentins par le roi d'Aragon en I404, évoquées par Goro Dati.

32. C'est le cas de trois ambassades rapportées par B. Pitti (au roi de France en I388, à l'antipape Benoît XIII en I395, à l'empereur en I4OI), de deux ambassades relatées par J. Salviati (au roi de France en I404, à Benoît XIII en 1407 ), tandis que B. Magalotti rapporte la participation d'un membre de sa famille à l'ambassade à Grégoire XI (1373) et que A. de' Ricci fait de même à propos de l'ambassade à l'empereur de 1352 et de l'ambassade au comte d'Armagnac de 139i. Notons que les récits d'ambassade de Pitti et Salviati, dont la décennie de référence est I4IO, comptent pour beaucoup dans le "pic» — très relatif — de notes concernant l'étranger qui apparaît sur les graphiques; cela correspond à une augmentation du nombre de récits d'ambassades qui concerne l'ensemble du corpus (dans comme hors de la Péninsule).

33. Cf. supra, note 29.

34. Naddo da Montecatini, qui paraît suivre de très près les décisions prises par la Seigneurie de Florence, rapporte deux ambassades au roi de France, en I386 — pour une affaire concernant aussi les Anjou-Duras et I389, ainsi que l'ambassade au comte d'Armagnac de I39I, qui s'inscrit dans le cadre de la lutte entre la Commune et le comte de Vertus.

35. Cf. supra, note 6.

36. B. Pitti, qui relate dans son livre son séjour en France (ouvr. cité, p. 379-392), consacre trois notes à la guerre de Cent Ans; S. Mannini, qui a résidé à Paris (ouvr. cité, p. 433 = fo 65), lui en consacre huit.

37. Si Naddo da Montecatini et G. Morelli la relatent sans être directement impliqués dans les faits, ce n'est pas le cas de B. Pitti, qui se présente systématiquement comme un acteur des événements. 
de Constantinople en I453 et la bataille de Belgrade en I456 - peuvent être considérées comme les témoins d'une ouverture sur l'Ailleurs qui ne soit pas "directement intéressée», même si l'on a vu qu'elles sont loin d'être totalement déconnectées de l'Ici.

En conclusion, il apparaît que la présence marginale de l'Orient dans les livres de famille florentins entre I30O et I 480 est non seulement cohérente avec les spécificités de cette forme d'écriture - directement liée à la défense de l'intérêt de la famille dans la cité - mais aussi avec le traitement de l'Ailleurs en général dans ces livres privés. L'étude des formes de cette présence, pour marginale qu'elle soit, a cependant permis de mettre en évidence deux éléments remarquables. Tout d'abord, l'existence chez les scripteurs, à partir des années I420, d'un sentiment d'appartenance élargi : alors qu'eux ou leurs familles ne participent pas de façon directe aux faits décrits, ils ressentent tout de même le besoin de les relater en vertu de leur appartenance à la communauté italienne et chrétienne. Le regard des Florentins sur eux-mêmes et sur le monde les entourant semble donc avoir connu une mutation aux alentours des années I420, dont il est frappant de constater qu'elle coïncide avec la mise en œuvre d'une politique volontaire d'ouverture de la Commune sur l'ailleurs, par le biais de sa flotte notamment. On peut évidemment se demander dans quelle mesure cette mutation des intérêts manifestés par les Florentins au sein de leur écriture privée fut influencée par les discours émis et portés par les autorités, ou par les ouvrages historiographiques qui circulaient alors, tels les Historiarum florentini populi libri XII de Leonardo Bruni, ce qui supposerait de comparer systématiquement le traitement des différents événements dans les sources privées et publiques.

\section{Bibliographie (hors manuscrits et thèses)}

Babinger Franz, "Lorenzo de' Medici e la Corte ottomana», Archivio storico italiano, vol. I2I, I963, p. 305-36I.

BeC Christian, Les livres des Florentins (I413-1608), Florence, Olschki, 1984. Capponi Gino, Storia della Repubblica di Firenze, II, Florence, Barbèra, I875.

Cardini Franco, Toscana e Terrasanta nel Medioevo, Florence, Alinea, 1982. Cardini Franco, In Terrasanta. Pellegrini italiani tra Medioevo e prima età moderna, Bologne, Il Mulino, 2002.

Castellani Francesco di Matteo, Ricordanze A (1436-I459), G. Ciappelli (éd.), Florence, Olschki, I992. 
Catellacci Dante, «Diario di Felice Brancacci ambasciatore con Carlo Federighi al Cairo per il Comune di Firenze», Archivio storico italiano, vol. 8, I88I, p. I57-I88; 326-334.

Cazalé-Bérard Claude et Klapisch-Zuber Christiane, «Mémoire de soi et des autres dans les livres de famille italiens ", Annales HSS, vol. 59, $\mathrm{n}^{\circ} 4,2004$, p. $805-826$.

Dei Benedetto, La cronica dall'anno I400 all'anno I50o, R. Barducci (éd.), Florence, Papafava, I984.

Del Corazza Bartolomeo di Michele, Quaderni overo Libro. Diario fiorentino (I405-I439), R. Gentile (éd.), Rome, De Rubeis Editore, I99I.

Del Piazzo Marcello (éd.), "Gli autografi di L. De’ Medici nell'Archivio di Stato di Firenze», Rinascimento, vol. 8, 1957, p. 213-260.

Dini Bruno, "L'economia fiorentina dal I450 al I530", dans Saggi su una economia-mondo. Firenze e l'Italia fra Mediterraneo ed Europa (secc. XIII-XVI), Pise, Pacini, I995, p. I87-2I4.

L'Europa dopo la caduta di Costantinopoli: 29 maggio I453 (Atti del XLVI convegno storico internazionale, Todi, octobre 2007), Spolète, CISAM, 2008.

Fiorato Adelin-Charles, "Propedeutica e legittimazione al potere nei "Ricordi" di Bonaccorso Pitti», dans L. Secchi Tarugi (éd.), Cultura e potere nel Rinascimento (Atti del IX Convegno internazionale, ChiancianoPienza, 1997), Florence, Franco Cesati Editore, p. 19-32.

GirARdi Raffaele, Raccontare l'Altro. L'Oriente islamico nella novella italiana da Boccaccio a Bandello, Naples, Liguori, 2012.

Hankins James, "Renaissance Crusaders: Humanist Crusade Literature in the Age of Mehmed II", Dumbarton Oaks Papers, vol. 49, I995, p. III-207.

Hankins James, Renaissance Civic Humanism: Reappraisals and Reflections, Cambridge, Cambridge UP, 2000.

Hoshino Hidetoshi, L'Arte della Lana in Firenze nel Basso Medioevo. Il commercio della lana e il mercato dei panni fiorentini nei secoli XIII-XV, Florence, Olschki, 1980.

Hoshino Hidetoshi, "I mercanti fiorentini ad Alessandria d'Egitto nella seconda metà del Trecento", dans Industria tessile e commercio internazionale nella Firenze del tardo Medioevo, F. Franceschi et S. Tognetti (éd.), Florence, Olschki, 200I, p. IOI-II2.

Hoshino Hidetoshi, «Il commercio fiorentino nell'Impero Ottomano: costi e profitti negli anni I484-I488", dans Industria tessile e commercio internazionale nella Firenze del tardo Medioevo, F. Franceschi et $S$. Tognetti (éd.), Florence, Olschki, 200I, p. II3-I23. 
Klapisch-Zuber, Christiane, La Maison et le nom. Stratégies et rituels dans l'Italie de la Renaissance, Paris, EHESS, I990.

Meserve Margaret, Empires of Islam in Renaissance Historical Thought, Cambridge Mass., Harvard UP, 2008.

Molmo Anthony et Sznura Franek, "Brighe, affanni, volgimenti di stato». Le ricordanze quattrocentesche di Luca di Matteo di messer Luca dei Firidolfi da Panzano, Florence, SISMEL-Edizioni del Galluzzo, 2010.

Mordenti Raul (dir.), I libri di famiglia in Italia, II, Rome, Edizioni di Storia e Letteratura, 200I.

MülLER Giuseppe, Documenti sulle relazioni delle città toscane coll'Oriente cristiano e coi turchi fino all'anno MDXXXI, Florence, Cellini, I879.

Naddo da Montecatini, Memorie storiche da un libro di ricordi scritto da Naddo di ser Nepo di ser Galo da Montecatini di Valdiniveole cittadino Fiorentino. Dall'anno 1374 all'anno I398, I. di San Luigi (éd.), Delizie degli eruditi toscani, XVIII, Florence, Cambiagi, I784, p. I-I7O.

Neri di Bicci, Ricordanze segnato D, B. Santi (éd.), Pise, Marlin, 1976.

Orvieto Paolo, "Un esperto orientalista del '400: Benedetto Dei», Rinascimento, vol. 9, I969, p. 205-275.

Pandimiglio Leonida, "Pigliate esempro di questo caso: l'inizio della scrittura di Bonaccorso Pitti", Lettere italiane, vol. 40, $\mathrm{n}^{\circ}$ 2, I988, p. I6I-I73.

Pertusi Agostino (éd.), La caduta di Costantinopoli. Testi, I-II, Milan, Mondadori, 1976.

Pezzarossa Fulvio, "La tradizione fiorentina della memorialistica», dans G. M. Anselmi, F. Pezzarossa et L. Avellini (éd.), La «memoria» dei mercatores. Tendenze ideologiche, ricordanze, artigianato in versi nella Firenze del Quattrocento, Bologne, Pàtron, 1980, p. 4I-I49.

Pitтi Bonaccorso, "Ricordi", dans V. Branca (éd.), Mercanti scrittori, Ricordi nella Firenze tra Medioevo e Rinascimento, Milan, Rusconi, I986, p. 343-503.

Ricci Giovanni, I Turchi alle porte, Bologne, Il Mulino, 2008.

Rucellai Giovanni, Zibaldone Quaresimale, A. Perosa (éd.), Londres, The Warburg Institute, 1960.

Schwoebel Robert, The Shadow of the Crescent: The Renaissance Image of the Turk (1453-I5I7), New York, St. Martin's Press, 1967.

Setton Kenneth M. (éd.), «The Emperor John VIII slept here», Speculum, vol. $33, \mathrm{n}^{\circ} 2$, 1958, p. 222-228.

Tanzini Lorenzo, «Il Magnifico e il Turco. Elementi politici, economici e culturali nelle relazioni tra Firenze e Impero Ottomano al tempo di Lorenzo de' Medici ", RiMe, no 4, 20IO, p. 27I-289. 
LE SPECTRE DE L'ORIENT DANS LES LIVRES DE FAMILLE FLORENTINS (I3OO-I480)

Tripodi Claudia, «Viaggi di ambasciatori tra Firenze e Il Cairo nel XV secolo", Mélanges de l'École française de Rome - Moyen Âge, vol. I22, $\mathrm{n}^{\circ}$ 2, 2OIO, p. 4II-440.

Velluti Donato, La cronica domestica di messer Donato Velluti scritta fra il 1367 e il 1370 con le addizioni di Paolo Velluti, scritte fra il 1555 e il Is60, I. del Lungo (éd.), Florence, Sansoni, I9I4. 